\title{
Reasoning with Imprecise Privacy Preferences
}

\author{
Inah Omoronyia \\ School of Computing Science \\ University of Glasgow, UK \\ inah.omoronyia@glasgow.ac.uk
}

\begin{abstract}
User reluctance and context-dependent factors during information disclosure imply that people cannot always be counted on to indicate their appropriate privacy preference. This phenomenon is the well-known 'privacy paradox', which shows that users of modern technologies are constantly concerned about their privacy, but do not apply these concerns to their usage behaviour accordingly. The problem is that this mismatch between privacy concerns and the indicated privacy preference in software, is not considered when reasoning about the satisfaction of privacy requirements.

This paper is a research vision that draws connections between the imprecisions in user privacy preferences, and reasoning about the satisfaction of privacy requirements. We outline the close relationship between privacy and user beliefs and uncertainties. We then propose a multi-agent framework that leverage on this relationship when reasoning about the satisfaction of privacy requirements. We anticipate that this vision will help reduce the gap between an increasingly complex information age and the software techniques needed to protect user privacy.
\end{abstract}

\section{CCS Concepts}

-Security and privacy $\rightarrow$ Software security engineering; •Software and its engineering $\rightarrow$ Requirements analysis;

\section{Keywords}

privacy, belief, uncertainty, requirements verification

\section{INTRODUCTION}

Social networking, internet-of-Things, cloud and mobile computing are examples of modern technologies that promise to reshape our daily activities. Underlying the use of these technologies is the seamless flow of information from one user or device to another. Such information may be personal, hence deserving appropriate privacy considerations.

Permission to make digital or hard copies of all or part of this work for personal or classroom use is granted without fee provided that copies are not made or distributed for profit or commercial advantage and that copies bear this notice and the full citation on the first page. Copyrights for components of this work owned by others than ACM copy otherwise, or republish, to post on servers or to redistribute to lists, requires prior specific permission and/or a fee. Request permissions from Permissions@acm.org.

FSE'16, November 13-18, 2016, Seattle, WA, USA

(C) 2016 ACM. 978-1-4503-4218-6/16/11 ...\$15.00

http://dx.doi.org/10.1145/2950290.2983982
In fact, various empirical studies have shown that the lack of appropriate privacy has seriously deterred users from the benefits of these technologies [11]. Also, the manner they invisibly collect, process and disseminate information in the midst of people's private lives has given rise to undesired consequences, such as non-acceptance and failure of new services, damage to reputation, or costly lawsuits [18].

In part, the problem with privacy in these technologies is that traditional software engineering techniques for identifying, analysing and verifying user privacy requirements are inadequate. Particularly, some of the challenges lie with user privacy preferences. First, the common practice in software design where users are presented with a privacy preference settings interface, via which they have to express their privacy requirements is too static and complex[1]. These settings are sometimes very difficult to understand, and seldom changed as users acquire new knowledge of adversaries, nor adapt as new privacy threats emerge. Indeed, solely relying on users to reconfigure their privacy settings when their context or preferences changes do not scale. For instance, Facebook has 61 privacy controls [3], and as at when Ugander et.al [15] carried out their study, an average Facebook user had about 190 friends. This already means that users will need to consider a huge space of potential privacy configurations. Thus, getting privacy setting right would pose a non-trivial task for even expert users.

Secondly, the problem with privacy preference is inherent in users themselves. This is because users rarely have a clear knowledge of what information other users have about them or how that information is used and with what consequences[2]. In other cases even when users are aware of the consequences of disclosure decisions, they remain unsure about their own preferences of how much privacy they want [12]. These problems are made worst by theoretical suggestions that if users are handed control of their information, they should be able to manage their privacy [1]. Yet empirical evidence suggests that users often lack the awareness and knowledge to set appropriate privacy preference[2].

Privacy preferences expressed by users are subsequently utilised by the software to determine the appropriate disclosure protocol that ensures the satisfaction of privacy requirements. There are many of such protocols. For example, in order to satisfy a privacy requirement, it may be essential that if information is requested by a recipient, then the sender is required to seek consent from the subject before the information is sent to the recipient. For other cases, a disclosure protocol may be that a granted consent is acknowledged by the sender, and the subject is notified when information 
is sent to the recipient [7]. Surely, there are finite ways of combining information request, consent, send and notice actions to define a disclosure protocol. The open question then is, given the fuzzy nature of privacy preferences, how can the extent of satisfaction of a privacy requirement be determined for a specific disclosure protocol. Indeed, the common assumption that by urging users to grant consent before information is disclosed always address their privacy needs is flawed. There may be scenarios where consent is granted, yet privacy is still violated [13]. In previous work [9][8], we highlighted that the identification of appropriate disclosure protocol that satisfies a privacy requirement, would also implore a reasoning mechanism that considers the imprecise nature of user privacy preferences.

To this end, we present a research vision of software solutions that can ameliorate the challenges of privacy preferences in modern technologies. To attain a better software engineering perspective of the problem void of lowlevel technical issues, we considered user interaction activities that ensue through modern technologies as informationflow networks. Where each node in the network is an agent representing a user of a device, a service provider or requester, and so on. Edges depict information-flow channels between agents enabling interactions. Above highlighted privacy problem is therefore reminiscent of a multi-agent setting where agents continually disclose personal information about the users they represent, and the difficulty of understanding the consequence of disclosure on the satisfaction of user privacy requirements. It is easy for information once disclosed to reach unintended agents, and it is frequently the case that users are unclear if an information-flow path will ultimately lead to privacy violation [18].

The motivations for representing users as agents in our abstraction are numerous. For instance, agent technology provides the technical infrastructure for automatic or semiautomatic communication and negotiation, which otherwise may be difficult to achieve by users in environment where context changes frequently.More importantly, agents can emulate many processes in social settings. For example the cooperative, learning, reactive and intentional properties of agents are similar to the way humans interact and exchange information in a social setting [17]. In a research trend paper, van Lamsweerde [16] even highlights the central role of agent-based reasoning in requirements analysis.

We now highlight three key contributions that this research would make to the state-of-the-art. First, we propose that utilising agents to assist a user in making appropriate information disclosure decisions can ameliorate the problems with privacy preference settings. While research into how user privacy can be enhanced using multi-agent systems is not new [14], state-of-the-art assumes that users know the appropriate disclosure protocols that preserve their privacy. Such assumption is not suitable for scenarios where users lack the awareness and knowledge to reason about the appropriateness of selected disclosure protocols. This research proposes a multi-agent approach where for a given privacy requirement and information-flow network, a subset of privacy preserving information-flow paths and associated disclosure protocols is recommended. Such mechanism could help users select appropriate disclosure protocols that maximise the satisfaction of privacy requirements.

The second research contribution is insights on the role of belief and uncertainty in reasoning about the satisfaction of user privacy requirements. A user beliefs a fact if it knows the fact. Whereas, uncertainty is a fact that can be known but not currently known by the user [5]. The level of such uncertainty may increase or reduce as information is shared in the network. While agent-based systems highlight the importance of knowledge sharing as a means to update agent beliefs[4], it remains unclear how such beliefs may be used to manage user privacy. However, the social perspective of using belief and uncertainty in reasoning about privacy occurs in many information disclosure settings. For instance, our belief of what others do or do not know could impact on our disclosure decision. Also, successive information processing steps over an information-flow path can change our beliefs, and this too is essential when deciding on most appropriate disclosure actions to take[8]. This research will investigate a model of agent belief and uncertainty that is suitable for privacy requirements analysis.

The final research contribution is a privacy requirement reasoning mechanism. As highlighted, imprecise privacy preferences makes it difficult to justify the absolute satisfaction of a privacy requirement. Rather, we are of the view that the satisfaction of privacy requirements cross-cuts a spectrum ranging from its being unsatisfied to fully satisfied. This is partly as a result of the contextual nature of user privacy. What is private in one context may not be private in another. Thus, to make appropriate disclosure decisions, a reasoning mechanism is needed to enable users to understand the extent of satisfaction of their privacy requirements. This view is similar to the notion of goal satisficing that is used in goal modelling to express requirements that cannot be satisfied in a clear-cut manner [6]. Our vision is to demonstrate that using certain disclosure protocols in a network enhances or abates the satisfaction of privacy requirements. This research will demonstrate how specific disclosure protocols may be incompetent for achieving desired levels of privacy requirement satisfaction.

In the remaining of this visionary paper, we present some insights on how privacy can be managed based on beliefs and uncertainty in section 2 . We then propose a reasoning framework central to realising our vision of privacy in section 3. We then finalise with conclusions in section 4

\section{PRIVACY AND KNOWLEDGE}

Our beliefs and uncertainties in knowledge are central to the manner we foster interactions in social settings. We coordinate and regulate our disclosure behaviour based on our beliefs about those we interact with and what information we want them to belief or be uncertain about. For instance, it's unlikely that during interaction with a work colleague to divulge intimate family information. This may be because we belief that the colleague should remain uncertain of such information to keep work relations cordial. But while at home, we may freely divulge such information to family members. In addition, the beliefs and uncertainty of users may transform over time. If a user was previously uncertain of a fact, a subsequent disclosure action may transform the knowledge of the user to belief in that fact. Conversely, it is practical to consider that a user's belief may at a later time transform to uncertainty. For instance, if the user becomes aware that his/her belief is predicated on false information. Indeed, a knowledge transformation from one form of uncertainty to another is also possible. Based on this view, there are two factors that can influence a user disclosure 


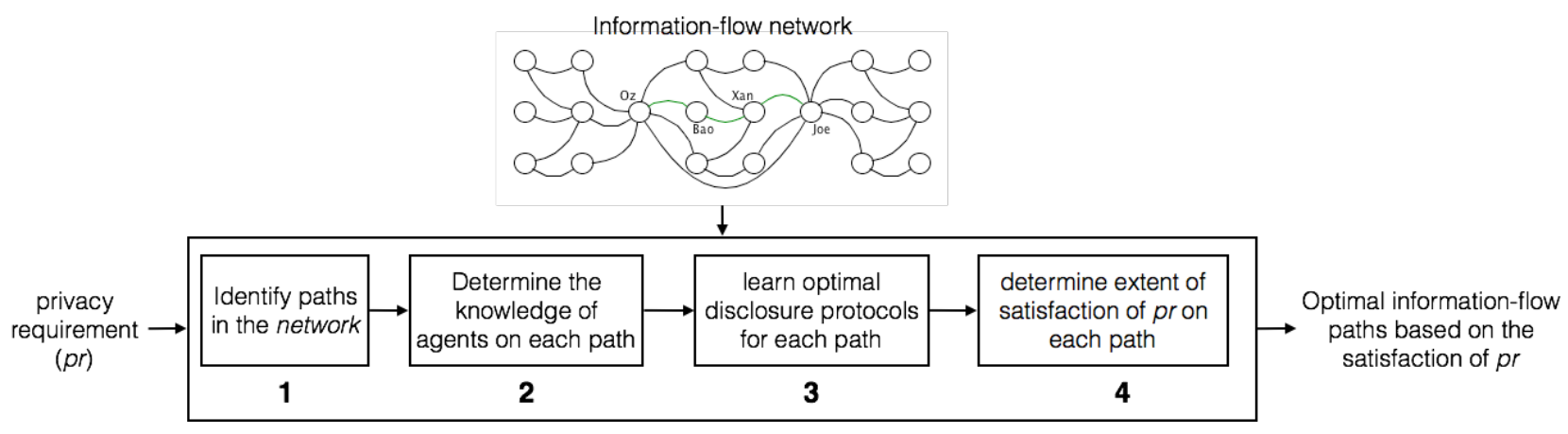

Figure 1: Proposed privacy reasoning framework

behaviour: (1) the user's current beliefs and uncertainty in knowledge, and (2) the desired belief and uncertainty that other users should have after disclosure.

Privacy is therefore the ability for a user to regulate the transformation of his/her belief and uncertainty, and that of others, during information disclosure. This view is consistent with modern description of privacy as informational self-determination [10]. Moreover, this view enables us to depict privacy as a balancing act on assurance and control. The assurance of privacy preservation is low when a user is uncertain of the knowledge that other users in a network will have as a result of disclosure. Whereas, control is the ability for a user to determine the extent other users in a network should be uncertain or belief a sensitive information on disclosure. The more uncertain other users are, the more private the information. Conversely, the more other users belief the information, the less private. A privacy requirement is then a specification of the level of assurance and control that a user should have. From an informationflow viewpoint, certain disclosure protocols may enhance or abate the satisfaction of a privacy requirement.

Reasoning about the satisfaction of a privacy requirement in multi-agent setting therefore entails a proper syntax and semantics for representing a user's belief and uncertainty. The representation mechanism should enable agent keep track of their belief and uncertainty states. Moreover, a disclosure protocol determines the manner that beliefs and uncertainties of agents are transformed. A protocol that includes a consent action when disclosing information about a subject, will transform the knowledge of the subject to belief that the sender knows that information. Whereas for a protocol without consent, the subject will be uncertain if the sender knows the information. Similarly, a protocol that notifies the subject after the information is sent to the recipient will transform the knowledge of the subject to belief that the sender knows that the recipient knows the information. Consequently, privacy requirements reasoning should also be amenable to dynamic belief and uncertainty transformation as information is disclosed from one agent to another.

\section{PRIVACY REASONING FRAMEWORK}

Our proposed framework for reasoning about the satisfaction of privacy requirements is shown in Figure 1. It takes as input a multi-agent information-flow network and the privacy requirement to be analysed. The output is a set of paths in the network that optimises the satisfaction of the privacy requirement. To streamline privacy analysis, the framework will enable the configuration of regions in the network where the privacy requirement will be verified. There are two ways such configuration could be achieved. The first involves specifying the depth of paths from a source agent that will be involved in privacy analysis. Assuming the input network in Figure 1 with agent $\mathrm{Oz}$ as the source. Then privacy analysis for depth $=2$ will involve 16 unique paths (each of the same length) and 17 agents. Using a social network as an example, such configuration will enable a user to insist that a privacy requirement should be satisfied to a level for all friends and friend of friends. Similarly, $1<\operatorname{depth} \leq 2$ will limit privacy analysis to only friend of friends.

The second option is to identify a specific target agent. The region of privacy analysis is then a set of paths from the source to selected target. This is useful when the desire is to gain insight on the extent of satisfaction of the privacy requirement with respect to a specific user agent. Again using the input network in Figure 1, and assuming Joe is the target agent of interest. Then, privacy analysis will involve all 10 unique paths of varying lengths from $\mathrm{Oz}$ to Joe. In both configuration approaches, the length of paths may be sufficiently long to scope the whole network, in which case privacy analysis will involve every agent in the network.

Once a region for privacy analysis in a network is configured and privacy requirement specified, the reasoning framework follows four steps to rank paths based on the extent they satisfy the privacy requirement. The first step is to identify paths from source to each target in the network. If the region of interest is specified by path depth, then paths from source to different targets are identified using k-nearest neighbour algorithm. Alternatively, when a region is based on a specific target agent, then a depth first search (DFS) is used. One problem with DFS is the likelihood of infinitely long paths for non-trivial networks. To address this problem, paths with cycles are not allowed.

The next set of steps involves reasoning about the extent of satisfaction of privacy requirements on each path. For the purpose of illustration, assuming $\mathrm{Oz}$ is to decide on disclosing information to Bao, and that the information may flow along the path $\mathrm{Oz} \rightarrow \mathrm{Bao} \rightarrow \mathrm{Xan} \rightarrow$ Joe. The source being $\mathrm{Oz}$ and target Joe. Then each consecutive pair along the path defines an information-flow towards the target. For the flow between $\mathrm{Oz}$ and Bao, there are two pertinent considerations that can assist $\mathrm{Oz}$ in making appropriate disclosure decision. The first is how Oz's beliefs and uncertainty about what Bao knows will be transformed after disclosure. Also, what new or transformed belief will Bao have about 
Oz. Secondly, would these new or transformed beliefs and uncertainties preserve 0z's privacy. What sort of disclosure protocol would $\mathrm{Oz}$ then need to in order to ensure that a level of privacy satisfaction is achieved. To gain insight on the whole path, same pattern of belief and uncertainty reasoning is also applied to each subsequent flow until the target is reached. If the overall privacy satisfaction across the path reaches an acceptable level, then disclosure along the path can be considered. Alternatively, disclosure is renegotiated or information not disclosed. Achieving this requires the framework to determine agent knowledge along each path.

Information disclosure along a path may increase or reduce the belief and uncertainty of associated agents. The next two steps in our framework will therefore investigate how agent disclosure behaviour may impact on the extent to which a privacy requirement is satisfied. We will model the behaviour of agents and ensuing beliefs and uncertainties using a suite of disclosure protocols. Furthermore, we will investigate novel options for representing and analysing the satisfaction of privacy requirements. We envision privacy requirements as a means to regulate the knowledge of agents in an information-flow network. It's an expression of the desired rate at which uncertainties are transformed to beliefs or other forms of uncertainty in a network (the socalled knowledge entropy). Alternatively, a privacy requirement highlights the level of belief or uncertainty that agents should have about disclosed information. Equally, a privacy requirement in its most granular form is an expression of specific belief and uncertainty elements that should constitute the knowledge of agents for privacy to be preserved. We will demonstrate how each of this representation and analytical approach can be used to determine optimal disclosure protocols along a path, and also generate a ranking of paths based on the extent a privacy requirement is satisfied.

\section{CONCLUSION}

We anticipate that our research vision sets the pace for a change in the way privacy is engineered in software systems. We argued that user privacy preferences may not be distinguishable in a clear-cut manner. Hence, any privacy requirements reasoning framework should factor in the imprecise nature of privacy requirements. We proposed a framework that regulates user beliefs and uncertainties to assist in such reasoning. Using our approach, disclosure decisions can be made dynamically, and informed by the runtime transformation in the belief and uncertainties of users. We expect that this will address current challenge of static and nonadapting privacy preference prevalent in the state-of-the-art. Furthermore, the inherent problem of lack of awareness is addressed by the pre-emptive properties of proposed framework. A key output of privacy reasoning is a prior insight on information-flow paths that may result in privacy violation if a user chooses to exhibit certain disclosure behaviour.

Generally, we are motivated by the view that with the current pace of technological evolution, the mechanisms for managing privacy in software are becoming obsolete. New privacy protection mechanisms need to be sufficiently flexible to evolve with the emerging unpredictable complexities of the information age. While we may not advocate for full privacy automation, there is a need for software to provide users with more insight into the consequences of information disclosure, as well as minimise the demands and expectations from users.

\section{ACKNOWLEDGMENTS}

This research is supported by EPSRC-ISF 2016 Institutional support grant award EP/P51133X/1

\section{REFERENCES}

[1] A. Acquisti, I. Adjerid, and L. Brandimarte. Gone in 15 seconds: The limits of privacy transparency and control. IEEE Security \& Privacy, 11(4):72-74, 2013.

[2] A. Acquisti, L. Brandimarte, and G. Loewenstein. Privacy and human behavior in the age of information. 347(6221):509-514, 2015.

[3] J. Bonneau and S. Preibusch. Economics of Info. Security and Privacy, chapter The Privacy Jungle:On the Market for Data Protection in Social Networks, pages 121-167. Springer US, Boston, MA, 2010.

[4] T. Finin, R. Fritzson, D. McKay, and R. McEntire. Kqml as an agent communication language. In International conference on Information and knowledge management, pages 456-463. ACM, 1994.

[5] M. Georgeff, B. Pell, M. Pollack, M. Tambe, and M. Wooldridge. The belief-desire-intention model of agency. In Intelligent Agents V: Agents Theories, Arch., and Languages, pages 1-10. Springer, 1998.

[6] J. Mylopoulos, L. Chung, and E. Yu. From object-oriented to goal-oriented requirements analysis. Commun. of the ACM, 42(1):31-37, 1999.

[7] H. Nissenbaum. Privacy in context: Technology, policy, and the integrity of social life. Stanford Uni. Press, 2009.

[8] I. Omoronyia. The case for privacy awareness requirements. Int. Jour. of Secure Software Eng., 2016.

[9] I. Omoronyia, L. Cavallaro, M. Salehie, L. Pasquale, and B. Nuseibeh. Engineering adaptive privacy: On the role of privacy awareness requirements. ICSE '13, pages 632-641, 2013.

[10] K. Rannenberg, D. Royer, and A. Deuker. The future of identity in the information society: Challenges and opportunities. Springer Sc. \& Business Media, 2009.

[11] M. D. Ryan. Cloud computing privacy concerns on our doorstep. Commun. ACM, 54(1):36-38, Jan. 2011.

[12] P. Slovic. The construction of preference. American psychologist, 50(5):364, 1995.

[13] D. J. Solove. Introduction: Privacy self-management and the consent dilemma. Harv. L. Rev., 126:1880, 2012.

[14] J. M. Such, A. Espinosa, and A. Garcia-Fornes. A survey of privacy in multi-agent systems. The Knowledge Engineering Review, 29:314-344, 62014.

[15] J. Ugander, B. Karrer, L. Backstrom, and C. Marlow. The anatomy of the facebook social graph. CoRR, abs/1111.4503, 2011.

[16] A. van Lamsweerde. Requirements engineering in the year 00: A research perspective. ICSE '00, pages 5-19, New York, NY, USA, 2000. ACM.

[17] M. Wooldridge and N. R. Jennings. Intelligent agents: Theory and practice. Knowledge Engineering Review, 10:115-152, 1995.

[18] J. H. Ziegeldorf, O. G. Morchon, and K. Wehrle. Privacy in the internet of things: threats and challenges. Security and Commun. Networks, 7(12):2728-2742, 2014. 